\title{
Apoyo directivo, consecuencia de carrera, y tiempo organizacional en el sector al por menor en Chile
}

\author{
Miguel A. Bustamante ${ }^{1 *}$, Andrés E. Jimenez ${ }^{2}$ y Melissa B. Contreras ${ }^{2}$ \\ (1) Facultad de Economía y Negocios, Universidad de Talca, Dos Norte 685, Talca, Chile. (Correo-e: mabu@utalca.cl) \\ (2) Facultad de Psicología, Universidad de Talca, Dos Norte 685, Talca, Chile. (Correo-e: anjimenez@utalca.cl; \\ melissa.contreras@utalca.cl) \\ * Autor a quien debe ser dirigida la correspondencia
}

Recibido Abr. 15, 2021; Aceptado Jun. 14, 2021; Versión final Jun. 30, 2021, Publicado Dic. 2021

\begin{abstract}
Resumen
El objetivo del presente trabajo fue determinar la relación entre apoyo directivo, consecuencia de carrera y demanda de tiempo organizacional en la conciliación trabajo-familia en el sector al por menor de Chile. A una muestra aleatoria de 618 personas se le aplicó un cuestionario estructurado en escala de cinco puntos. Se ejecutó un análisis factorial exploratorio y se modelaron las dimensiones mediante análisis estructural confirmatorio. Se determinaron dos factores de apoyo directivo y un factor de consecuencia de carrera con cargas factoriales altas y significativas. Los indicadores de varianza y covarianza resultaron positivos e idénticos entre los factores apoyo directivo. Sin embargo, resultaron ser negativos entre los factores apoyo directivo y consecuencias de carrera. Se concluye que estas variables actúan reduciendo los efectos adversos sobre las consecuencias de carrera, con lo cual se puede inferir que se reducen las eventuales tendencias de conflicto trabajo-familia.
\end{abstract}

Palabras clave: equilibrio trabajo-familia; apoyo directivo; desarrollo; carrera; gestión; recursos humanos

\section{Management support, career development, and time management in the retail sector in Chile}

\begin{abstract}
The present study aims to determine the relationships between managerial support, career development, and company's time demand and their impact on work-family balance in the Chilean retail sector. A random sample of 618 people is surveyed with a structured questionnaire on a five-point scale. An exploratory factor analysis is performed and dimensions are modeled by applying a confirmatory structural analysis. High and significant factor loadings are determined by using two managerial support factors and one career development factor. The results show that variance and covariance indicators are positive and identical between managerial support factors, but they are negative between managerial support and career development factors. It is concluded that these variables reduce adverse effects on career development, suggesting that there is a reduction in work-family conflict.
\end{abstract}




\section{INTRODUCCIÓN}

La cultura trabajo-familia, como relación conceptual multidimensional ha sido estudiada desde diversas perspectivas (Amstad et al., 2011; Pérez et al., 2017) relacionando el contexto de trabajo con los diversos roles familiares que hombres y mujeres desarrollan más allá de sus desempeños laborales (Warren y Johnson, 1995; Allen, 2001;). Este constructo relacional busca conciliar la complejidad del quehacer laboral con la vida de familiar en sus diversas dimensiones (Thompson et al., 1999) y pone de manifiesto que la relación trabajo y familia agudiza tensiones si las jornadas son extensas e impide a los trabajadores compatibilizar vida laboral con los tiempos requeridos por la familia (Jansen et al., 2010; McMillan et al., 2011). Dichas tensiones reducen la disposición de las personas a integrar ambos dominios porque las presiones provenientes de uno pueden dificultar la ejecución del otro y porque ambas dimensiones interactúan en forma bidireccional (Martínez-Pampliega et al., 2019). Es por ello que diversos estudios buscan explicar cómo una correcta gestión de las prestaciones familiares, arreglos flexibles y tiempos de trabajo, incrementan las oportunidades organizacionales y reducen el conflicto trabajo - familia (Jansen et al., 2010; Mauno et al., 2005; Jimenez-Figueroa y Bustamante, 2020) activando conductas de motivación laboral positiva que contrarrestan tensiones y se expresan en un mejor clima interno en las organizaciones (Bustamante y Álvarez, 2019).

La literatura presenta una variedad de estudios de la conciliación trabajo-familia dando pie a diversas aproximaciones las cuales impactan desde el nivel de operaciones pasando por el rol de supervisores hasta llegar a nivel directivo (Allen, 2001; Clark, 2001; McDonald et al., 2005). Las actividades del trabajo y de la familia inciden mutuamente vinculando ambas realidades, en palabras de Casper y Harris (2008), genera un sentido de identidad social de clara interferencia laboral que contrapone trabajo y familia (Pérez et al., 2017; Martínez-Pampliega et al., 2019;). Sin embargo, tal como lo demuestra Lapierre y Allen (2006), la flexibilidad temporal, operacional y el respaldo de los supervisores en relación con las responsabilidades familiares, permiten evitar el conflicto trabajo - familia (Jansen et al., 2010; Sivatte y Gauadamillas, 2014) compatibilizando la interfaz de dichos espacios e incrementando el desempeño de las organizaciones (Chinchilla et al., 2003; Frye y Breaugh, 2004). De esta forma estos enfoques se integran a un conjunto de variables significativas que potencian, por una parte, la lealtad y auto gestión de las iniciativas laborales por parte de los trabajadores (Smith y Gardner, 2007) al grado que las personas llegan a identificarse con la organización, particularmente si los trabajadores realizan sus labores en un contexto de trabajos flexibles (Litchfield et al., 2004).

En general, las dimensiones de la cultura trabajo - familia incluyen las relaciones de género en un mercado laboral que requiere talentos y capacidades diferenciadas, por lo mismo, parece tener sentido afirmar que se trata de armonizar un sistema complejo de apoyo parental significativo (Lapierre y Allen, 2006; Allard et al., 2011) gestionando horas de trabajo y conflictos familiares, por ejemplo, a nivel de directivos (Chinchilla et al., 2003; Frye y Breaugh, 2004), quienes, desde sus posiciones de ejecutivas y que en este trabajo se analizan a través de las variables de apoyo directivo, buscan mitigar incompatibilidades entre la vida familiar y el trabajo de sí mismos y de sus dirigidos (Warren y Johnson, 1995; Allen, 2001; Mauno et al., 2005). La gestión de las dimensiones de la conciliación trabajo - familia se manifiesta a través de múltiples formas, como por ejemplo, mediante programas de vida laboral con horarios flexibles (Clark, 2001) así como potenciando el trato preferencial para que los propios trabajadores en el ejercicio de su autonomía gestionen su bienestar y con ello logren consolidar los efectos positivos sobre el factor consecuencia de carrera aquí estudiado (Thompson y Prottas, 2005; García, 2019). Desde esta perspectiva, en el contexto brasileño, se observa cómo el apoyo directivo de los supervisores colabora positivamente y logra conciliar trabajo-familia (Casper et al., 2011; McMillan et al., 2011) cuando se potencian iniciativas de equilibrio, primero a causa del apoyo directivo y luego mediante la gestión de las dimensiones que conforman las demandas de tiempo organización (Thompson et al., 1999 Chinchilla et al., 2003; Smith y Gardner, 2007). En consecuencia, la gestión de las variables flexibilidad laboral, estilos de supervisión y apoyo directivo (Smith y Gardner, 2007) demuestran ser factores positivos en la dualidad trabajo - familia, de modo que si se gestionan con el apoyo gerencial, el clima interno de las organizaciones es positivo y las personas se motivan (Clark, 2001; Amstad et al., 2011; Bustamante y Álvarez, 2019) lo que incide positivamente sobre roles y tiempos de trabajo reduciendo el conflicto trabajo familia (Allen, 2001; Jansen et al., 2010; Pérez et al., 2017).

Así por ejemplo, la conciliación trabajo - familia analiza percepciones de bienestar laboral cuando supervisores y directivos cumplen una función amortiguadora del estrés y la tensión (McDonald et al., 2005; Lapierre y Allen, 2006; Casper et al., 2011), lo que resulta especialmente relevante si se busca reducir el conflicto y obtener los beneficios de la vida y del trabajo a partir de los intereses propios (Casper y Harris, 2008; Jansen et al., 2010; Jimenez-Figueroa y Bustamante, 2020) y que se consigue fortaleciendo la satisfacción laboral, el apoyo directivo y las conductas de auto cuidado, mismas que fortalecen el desempeño en el mundo del trabajo y facilitan la conciliación trabajo - familia (Chinchilla et al., 2003; Dawley et al., 2010; García, 2019). Es por ello que se requiere del esfuerzo de trabajadores, supervisores o directivos para gestionar las incompatibilidades trabajo - familia, por una parte, activando el apoyo de directivo y por otra, gestionando las 
demandas de tiempo organización, que conceptualmente por sí solas o en forma conjunta, inciden sobre la dimensión consecuencias de carrera (Warren y Johnson, 1995; Allen, 2001; García, 2019).

Sobre la base de los conceptos analizados, el objetivo del presente trabajo es analizar la conformación de las fuerzas de equilibrio trabajo - familia, determinando las relaciones que se producen entre las variables de apoyo directivo, demandas de tiempo organización y consecuencia de carrera en el sector de venta al por menor, retail, en Chile.

\section{METODOLOGÍA}

El presente estudio fue desarrollado sobre la base de los trabajos teóricos de Thompson et al., (1999) respecto de la conciliación trabajo-familia, que en esta ocasión se enfoca sobre tres de las dimensiones factoriales del modelo original. En consecuencia y sobre la base de las relaciones conceptuales que se producen entre las dimensiones, en este trabajo se analizan los factores a) apoyo directivo en relación con la dimensión b) consecuencias de carrera y con la dimensión c) demanda de tiempo organización (McDonald et al., 2005), a partir de lo cual se define la siguiente hipótesis de incidencia: H: que las prácticas organizacionales de la dimensión apoyo directivo inciden de manera inversa sobre la dimensión consecuencia de carrera y que el factor demanda de tiempo organización incide de manera directa sobre consecuencia de carrera. Consecuente con lo indicado, el objetivo del presente trabajo es establecer un modelo que determine la relación y dirección del efecto del apoyo directivo y demanda de tiempo organización sobre consecuencia de carrera de los personales del sector de venta al por menor, retail, en Chile.

Asumiendo el propósito declarado en la hipótesis $\mathrm{H}$, el estudio es no experimental, cuantitativo, transversal y exploratorio (Hernández-Sampieri y Mendoza, 2018) y luego relacional y causal para el análisis de un sistema de factores de la conciliación trabajo - familia buscando establecer relaciones estadísticamente validadas. Para ello se realizó el análisis factorial exploratorio de los reactivos seguido de un análisis confirmatorio de los constructos, todo ello en coherencia con la teoría referenciada (Thompson et al., 1999; McDonald et al., 2005). Luego se modelaron los factores de apoyo directivo, demandas de tiempo organización y consecuencias de carrera mediante el método de ecuaciones estructurales.

\section{Población y muestra}

La población en estudio quedó constituida por trabajadores del sector retail de 14 de las 15 regiones de Chile, todos adultos de entre 18 y 65 años. La muestra determinada a priori (Gpower 3.1.9.2.), previó una varianza máxima de $50 \%$ considerando una distribución normal de la población. Se contempló un intervalo de confianza de $95 \%$ ( 1 - b probabilidad de error) equivalente a una longitud aproximada $Z=1,96$, una pendiente $\mathrm{H} 1 \mathrm{de}$ 0,15 y un error de estimación de $5 \%$ alcanzándose una muestra representativa estimada de 472 unidades. El plan de muestreo de tipo probabilístico incluyó a empleados, directivos, profesionales, técnicos, administrativos y auxiliares (Hernández-Sampieri y Mendoza, 2018). Además, dado que la muestra supera tanto el criterio de muestra mínima $(n \geq 300)$ como el criterio de personas por ítem (N/p) (10:1 y 5:1) que determina el número de casos necesarios por cada uno de los reactivos del instrumento (Timmerman y Lorenzo-Seva, 2011; Freiberg et al., 2013), se puede asumir que el estudio supera los criterios requeridos para la obtención de resultados satisfactorios.

\section{Procedimiento}

El presente trabajo es el resultado de un proyecto de investigación previamente aprobado por el comité de ética de la Universidad de Talca que aprobó el instrumento de recogida de datos y los procedimientos de análisis previstos. Se optó por realizar el cuestionario en forma anónima bajo el principio de consentimiento informado (Hernández-Sampieri y Mendoza, 2018), considerando un método de aplicación en modalidad online, asegurando informar a cada entrevistado las bases y los términos de anonimato y confidencialidad respectivos. Seguidamente, el análisis de los datos se realizó resguardando la identidad, tratando de manera agrupada y con absoluta reserva la información de los respondientes. Además, con el propósito de asegurar calidad en la recogida de datos, evitar el estrés de los entrevistados y dada la envergadura del instrumento, la duración promedio de respuesta se estimó en aproximadamente 25 minutos.

\section{Descripción del instrumento}

El Cuestionario de Cultura Trabajo-Familia fue elaborado por Thompson et al., (1999), consta de 20 ítems y su formato de respuesta es una escala likert de 7 puntos donde el 1 representa totalmente en acuerdo y el 7 representa totalmente en desacuerdo. En sus detalles, el cuestionario mide tres componentes de la cultura trabajo-familia: Apoyo Directivo (AP), Consecuencias de Carrera (CC) y Demandas de Tiempo (DTO). La confiabilidad general alcanza un índice de 0,86, en tanto que Apoyo Directivo (11 ítems) fue de 0,85; de 0,79 para Consecuencias de Carrera (5 ítems) y de 0,76 para Demandas de Tiempo Organización (4 ítems). En consecuencia a partir de la hipótesis y objetivo declarados, se analiza en este trabajo la incidencia de la dimensión apoyo directivo, demanda de tiempo organización sobre consecuencia de carrera. 


\section{Análisis factorial y modelamiento estructural}

Dado que en el presente trabajo se analizan tres de las dimensiones del constructo de conciliación trabajo familia, cada una de ellas compuesta por un conjunto de variables observables diversas, se optó por aplicar el método de análisis factorial exploratorio (AFE), como alternativa semiconfirmatoria (Timmerman y LorenzoSeva, 2011) y con el objetivo de ratificar las relaciones e incidencias que se presenten entre los factores latentes contrastados. Adicionalmente se señala que el análisis factorial exploratorio, permite descubrir la estructura interna de relaciones entre un número relativamente alto de variables, para luego ser sometido a análisis factorial confirmatorio, mediante análisis estructural como el que más adelante esteb trabajo aplica mediante los estudios de varianza y covarianza que permiten aceptar o rechazar la hipótesis de relación o incidencia propuesta (Freiberg et al., 2013).A partir de lo señalado respecto del análisis factorial, se siguieron las fases recomendadas en la literatura y se optó por aplicar análisis paralelo (PA) mediante la matriz de dispersión policóricas (Timmerman y Lorenzo-Seva, 2011; Freiberg et al., 2013), análisis robusto de los mínimos cuadrados no ponderados (RULS) y rotación oblimin directo, puesto que permite asumir relaciones de interdependencia entre ítems cualitativos y factores latentes que pueden resultar correlacionados (Freiberg et al., 2013).

Seguidamente, para medir las percepciones de los entrevistados sobre los reactivos asociados a cada uno de los factores del modelo conceptual analizado (Freiberg et al., 2013) se utilizó la escala 1:7 la que dado su rango, permite asumir que se pueden dar por superados los supuestos de no normalidad de las escalas además de poder relacionar variables latentes que pudiesen pasar inadvertidas si se aplicara rotación ortogonal (Timmerman y Lorenzo-Seva, 2011). Así mismo, siguiendo las orientaciones técnicas para el análisis estructural, se optó por comunalidades altas $(>0,4)$, asegurando correlaciones directas y fuertes entre ítems y constructos (Timmerman y Lorenzo-Seva, 2011; Freiberg et al., 2013). Además se exigieron cargas factoriales también altas $(>0,4)$ con el propósito de excluir reactivos con baja correlación. Finalmente, se evalúo la confiabilidad de cada uno de los constructos mediante alfa de Cronbach y Omega de McDonald (Freiberg et al., 2013), se utilizó el índice de error raíz cuadrático medio, RMSR, la verificación del número de ítems mínimo (3) para cada constructo, la medida de adecuación $\mathrm{KMO}(\geq 0,80)$ con sus respectivos coeficientes de significancia de Bartlett y se analizó la varianza explicada para obtener configuraciones interpretables (Freiberg et al., 2013).

El Modelamiento Estructural se realizó previa revisión de las recomendaciones de Baño y Kaplan (Freiberg et al., 2013), se verificó la existencia de casos atípicos con valores extremos o poco significativos para asegurar que los resultados e interpretación de las estimaciones multivariantes fuesen pertinentes. Para confirmar ítems, se sensibilizaros las variables como policóricas mediante el programa Factor aplicando análisis factorial exploratorio (AFE). Luego se modelaron las variables latentes mediante análisis estructural, ratificado a través de los análisis de bondad de ajuste considerando los índices absolutos Estadístico-Radio de Verosimilitud de Chi-Cuadrado (CMIN/DF $\leq 3$ ), el Índice GFI $(\geq 0,9)$ que mide la variabilidad explicada por el modelo, el error de aproximación cuadrático medio $\operatorname{RMSEA}(\leq 0,05)$ y el índice de error cuadrático medio $\operatorname{RMR}(\approx 0)$. Además se verificaron las medidas de ajuste Comparative Fit Index (CFI $>0,9)$, Goodness of Fit Index $(\mathrm{GFI}>0,9)$, incremental Adjusted Goodness of Fit Index (AGFI>0,8), Goodness of Fit Index without diagonal values (GFI>0,8) (Timmerman y Lorenzo-Seva, 2011; Freiberg et al., 2013). Por último, el procesamiento de datos se realizó a través de los programas: SPSS (v.21), Gpower (3.1.9.2.), Factor (v.10.5.03) y AMOS (v.21) para Windows.

\section{RESULTADOS}

Se presentan de manera sintética los resultados del análisis descriptivo de la muestra, el análisis factorial y el modelamiento estructural respectivo.

\section{Análisis descriptivo de la muestra}

El instrumento logró determinar una muestra de 618 personas, superior a la muestra estimada (472), constituida por trabajadores mayores de edad (entre 18 y 65 años). De los 618 contactos, 441 son mujeres $(71,4 \%)$ y 117 son hombres $(28,6 \%)$. Sus edades se encuentran en un rango de 21 a 65 años, cuyo promedio general es de 38. La mayoría de los trabajadores (93,5\%) tiene un contrato de trabajo indefinido y con jornada completa $(95,2 \%)$. En nivel de estudios la mayor parte presenta educación universitaria completa $(42,6 \%)$ seguida de educación media incompleta $(29,2 \%)$. En estado civil prevalecen los trabajadores casados con un $36,7 \%$, quienes viven en pareja alcanzan a $17,6 \%$ y separados un $6,65 \%$. En general, el $66,1 \%$ señala ser el principal proveedor de su respectivo hogar. Adicionalmente, un $25,4 \%$ de los empleados señalan percibir ingresos entre $\$ 975.001$ - $\$ 1.575 .000$ (US\% 1.393 - US $\$ 2.250$ ) seguido de un $21,2 \%$ que se ubica en el rango de $\$ 300.001$ y $\$ 450.000$ (US\$ 429 - US\% 643). Por último, el $14,7 \%$ señala estar a cargo de un adulto mayor y el $69,7 \%$ tiene hijos a los que cuidar, en tanto que, entre quienes requieren de ayuda, tan solo el $12,5 \%$ cuenta con servicio doméstico a tiempo completo o parcial. 


\section{Análisis factorial exploratorio}

Como se observa en la Tabla 1, los tres constructos analizados a decir, apoyo directivo, consecuencia de carrera y demanda de tiempo organización presentan índices KMO adecuados de 0,92769, 0,73895 y 9,67428 respectivamente. Seguidamente los índices de confiabilidad Alfa de Cronbach y Omega de Mc Donald resultaron altos para los tres constructos $(0,69$ y 0,89$)$. Consecuentemente los constructos presentan además índices de bondad de ajuste CFI, y AGFI elevados que se ubican, en todos los casos, por encima del estándar requerido $(\geq 0,9)$. Finalmente, los índices de error RMSR y RMSA ratifican los factores determinados con índices alrededor de los valores estándar esperados $(\approx 0,05)$.

Tabla 1: Factores de apoyo directivo, consecuencia de carrera y demanda tiempo organización

\begin{tabular}{|c|c|c|c|c|c|c|c|c|c|c|}
\hline \multicolumn{4}{|c|}{ Apoyo directivo } & \multicolumn{3}{|c|}{ Consecuencia de carrera } & \multicolumn{3}{|c|}{$\begin{array}{c}\text { Demanda tiempo } \\
\text { organización }\end{array}$} & \multirow{2}{*}{$\begin{array}{l}\text { Variables } \\
\text { eliminadas }\end{array}$} \\
\hline VAR. & $A P 1$ & $A P 2$ & Comunalidad & VAR. & $C C 1$ & Comunalidad & $V A R$. & $\begin{array}{c}\text { DTO } \\
1\end{array}$ & Comunalidad & \\
\hline V 1 & 0,845 & & 0,583 & $\mathrm{~V} 12$ & 0,556 & 0,276 & V 17 & 0,437 & 0,423 & V17 \\
\hline V2 & 0,798 & & 0,782 & V 13 & & 0,410 & V 18 & 0,544 & 0,337 & V13 \\
\hline V 3 & 0,532 & & 0,584 & V 14 & 0,533 & 0,481 & V 19 & 0,729 & 0,531 & \\
\hline V 4 & 0,481 & & 0,419 & & & & V 20 & 0,699 & 0,807 & V 4 \\
\hline V 5 & 0,673 & & 0,641 & $\mathrm{~V} 15$ & 0,000 & 0,771 & & & & V15 \\
\hline V 6 & $-0,423$ & & 0,389 & & & & & & & V6 \\
\hline V 7 & 0,489 & & 0,508 & V 16 & 0,579 & 0,320 & & & & \\
\hline V 8 & & 0,416 & 0,644 & & & & & & & \\
\hline V 9 & & 0,695 & 0,587 & & & & & & & \\
\hline V10 & & 0,822 & 0,754 & & & & & & & \\
\hline V11 & 0,575 & & 0,687 & & & & & & & \\
\hline Sig. & $58,6^{*}$ & 8,1 & & & $62,3^{*}$ & & & $69,8^{*}$ & & \\
\hline \multicolumn{3}{|c|}{$\begin{array}{l}\text { Kaiser-Meyer-Olkin } \\
\text { KMO) }\end{array}$} & 0,928 & & & 0,739 & & & 0,674 & \\
\hline \multicolumn{3}{|c|}{$\begin{array}{l}\text { İndice de ajuste } \\
\text { comparativo (CFI) }\end{array}$} & 0,984 & & & 1,000 & & & 0,953 & \\
\hline \multicolumn{3}{|c|}{$\begin{array}{l}\text { Indice ajustado de } \\
\text { bondad de ajuste (AGFI) }\end{array}$} & 0,993 & & & 0,991 & & & 0,982 & \\
\hline \multicolumn{3}{|c|}{ a Cronbach } & 0,892 & & & 0,709 & & & 0,691 & \\
\hline \multicolumn{3}{|c|}{$\Omega$ McDonald's } & 0,896 & & & 0,715 & & & 0,699 & \\
\hline \multirow{2}{*}{\multicolumn{3}{|c|}{ RMSR }} & 0,0351 & & & 0,0076 & & & 0,0537 & \\
\hline \multicolumn{2}{|l|}{ RMSA } & & 0,073 & & & $\leq 0,05$ & & & 0,139 & \\
\hline
\end{tabular}

Para la dimensión de apoyo directivo, se determinaron dos factores (AP1 y AP2). El factor AP1 concentra seis (6) reactivos $(\mathrm{V} 1, \mathrm{~V} 2, \mathrm{~V} 3, \mathrm{~V} 5, \mathrm{~V} 7$ y $\mathrm{V} 11)$ correlacionados en rangos altos $(\geq 0,489)$ que se expresa en cargas factoriales superiores alcanzando una varianza explicada de $58,6 \%$ significativa $\left({ }^{*}\right)$. El factor AP2 conformado por tres (3) reactivos presenta una varianza explicada de 8,1\%, que sin alcanzar significación, permite que la dimensión apoyo directivo totalice $66,7 \%$ de varianza total explicada. Se aprecia así mismo que se excluyen de este constructo dos reactivos (V4 y V6) por cuanto las cargas factoriales y la comunalidad resultaron inferiores al mínimo requerido $(\geq 0,4)$. La dimensión consecuencia de carrera concentra tres (3) reactivos (V12, V14, V16) en un factor (CC1) que explica el $62,3 \%$ de la varianza explicada en forma significativa $\left(^{*}\right)$. Se excluyen en este caso dos (2) reactivos (V13 y V15), el primero porque tanto su carga factorial como su comunalidad resulta inferior al mínimo exigido $(\geq 0,4)$ y el segundo porque no presenta carga factorial aunque su comunalidad fue alta $(0,771)$. Complementariamente demanda de tiempo organización (DTO1) congrega tres (3) reactivos (V18, V19, V20) que explican un $69,8 \%$ de la varianza explicada también significativa $\left({ }^{*}\right)$. Esta última dimensión excluye un (1) reactivo (V17) por cuanto sus índices de carga factorial y comunalidad determinadas resultan inferiores al estándar exigido $(\geq 0,4)$.

\section{Modelamiento estructural}

Sobre la base de los componentes determinados, se realizó el modelamiento estructural aplicando los procedimientos de covarianzas y varianzas como se muestra en la Fig. 1. 

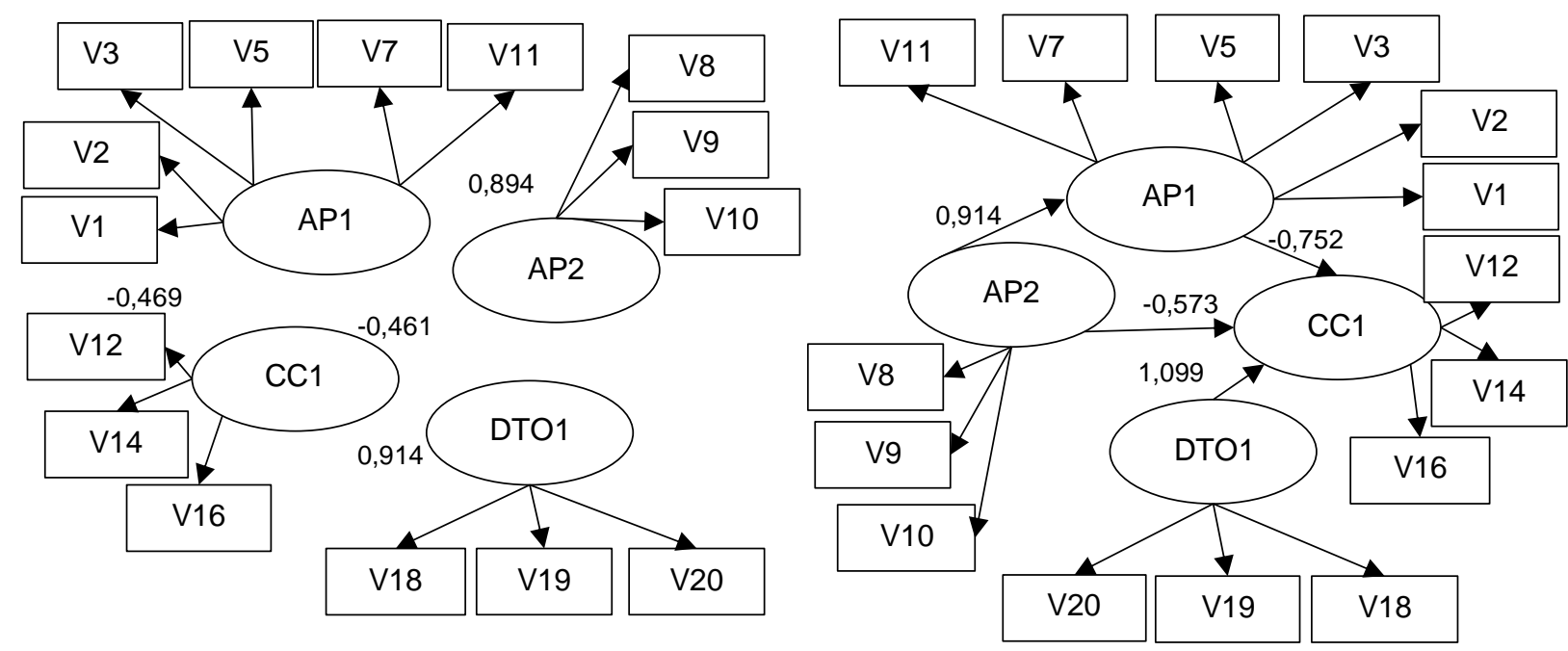

Figura 1: Modelamiento de covarianza y varianza de apoyo directivo, demanda tiempo organización y consecuencia de carrera.

En general se aprecia que los indicadores de covarianza y de varianza muestran índices de magnitud similar para la relación de covarianza y varianza que se produce entre los factores de apoyo directivo AP1<-->AP2 $(0,894 ; 0,914)$, lo cual indica que un incremento en un punto de uno de los factores incide directamente sobre el otro. Por otra parte, las relaciones de covarianza y varianza que se presentan entre AP1<-->CC1 son inversos y cercanamente similares $(-0,469$ y $-0,752)$ entre apoyo directivo y consecuencia de carrera, en consecuencia un incremento en un punto en el primer factor incide inversamente sobre el factor relacionado. Seguidamente, las relaciones de covarianza y varianza que se presentan entre estos factores AP2<-->CC1 son inversos y cercanamente similares $(-0,461$ y $-0,573)$ entre los factores AP2 y CC1, en consecuencia un incremento en un punto en el primer factor incide inversamente sobre el factor relacionado. En consecuencia, en esta fase general de análisis se aprecia que las relaciones de covarianza y varianza entre el factor consecuencia de carrera sobre demanda de tiempo organización $\mathrm{CC} 1<-->\mathrm{DTO} 1$ es positiva alcanzando índices relativamente cercanos en sus efectos $(0,914$ y 1,099), demostrando que estas relaciones son directas entre los factores consecuencia de carrera, CC1 y demanda de tiempo organización, DTO1 (Fig. 1). La tabla 2 muestra los estimadores y valores estándar del análisis estructural de las dimensiones de apoyo directivo, consecuencia de carrera y demanda de tiempo organización presentando estimadores estándar correctamente determinados (0-1).

Tabla 2: Modelo de medida de apoyo directivo, consecuencia de carrera y demanda de tiempo organización

\begin{tabular}{|c|c|c|c|c|c|c|c|c|}
\hline \multicolumn{2}{|c|}{ Análisis de varianza } & \multicolumn{2}{|c|}{ Apoyo directivo } & \multicolumn{2}{|c|}{ Análisis de covarianza } & \multirow{2}{*}{\multicolumn{3}{|c|}{$\begin{array}{c}\text { Relaciones del modelo } \\
\text { Varianzas }\end{array}$}} \\
\hline AP1 & $\begin{array}{l}\text { Estimador } \\
\text { Estándar }\end{array}$ & $P$ & AP1 & $\begin{array}{l}\text { Estimador } \\
\text { Estándar }\end{array}$ & $\mathrm{P}$ & & & \\
\hline V1 & 0,721 & & & 0,731 & $* \star \star$ & Variables & Estimador Estándar & $\mathrm{P}^{* \star *}$ \\
\hline V2 & 0,827 & $* * *$ & & 0,828 & $* \star *$ & AP1<--- AP2 & 0,914 & $* * *$ \\
\hline V3 & 0,569 & *** & & 0,571 & $\star \star \star *$ & CC1<--- DOT1 & 1,099 & 0,996 \\
\hline V5 & 0,799 & *** & & 0,808 & $* * *$ & CC1<--- AP1 & 0,752 & ** \\
\hline V7 & 0,549 & *** & & 0,560 & 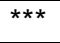 & CC1<--- AP2 & $-0,573$ & ** \\
\hline V11 & 0,793 & $* \star *$ & & 0,840 & $* \star *$ & & & \\
\hline AP2 & & & & AP2 & & & & \\
\hline V8 & 0,837 & & & 0,832 & $* * *$ & & & \\
\hline V9 & 0,716 & $\star * *$ & & 0,720 & $* * *$ & & & \\
\hline V10 & 0,772 & 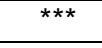 & & 0,775 & $\star \star \star$ & & & \\
\hline $\begin{array}{l}\text { DTO } \\
1\end{array}$ & \multicolumn{3}{|c|}{ Demanda tiempo organización } & DTO1 & & & & \\
\hline V18 & 0,491 & & & 0,491 & $* \star *$ & \multicolumn{3}{|c|}{ Relaciones del modelo } \\
\hline V19 & 0,662 & *** & & 0,662 & $* * *$ & \multicolumn{3}{|c|}{ Covarianzas } \\
\hline V20 & 0,853 & $* * *$ & & 0,853 & $* \star *$ & Variables & Estimador Estándar & \\
\hline CC1 & \multicolumn{3}{|c|}{ Consecuencia de Carrera } & CC1 & & AP1<--- AP2 & 0,894 & *** \\
\hline V16 & 0,559 & 0,996 & & 0,559 & $* * *$ & CC1<--- DOT1 & 0,914 & *** \\
\hline V14 & 0,700 & 0,996 & & 0,700 & $* * *$ & CC1<--- AP1 & $-0,469$ & *** \\
\hline V12 & 0,450 & 0,996 & & 0,449 & $* \star \star$ & CC1<--- AP2 & $-0,461$ & *** \\
\hline
\end{tabular}


En primer lugar, el análisis de covarianza permitió determinar, para la totalidad de los reactivos, estimadores estandarizados significativos $\left.{ }^{* * *}\right)$. En segundo lugar, el análisis de varianza muestra que los reactivos alcanzaron, en su mayoría, estimadores también significativos $\left({ }^{* * *}\right)$, destacándose por el contrario dos reactivos (V1 y V18) que resultan no significativos. Además, se aprecia en la Tabla 2, que resulta significativa $\left(^{* * *}\right)$ la relación de los factores de apoyo directivo (AP1<--- AP2), sin embargo, las relaciones cruzadas de los demás factores CC1, AP1 y AP2, aunque presentan estimadores adecuados resultaron significativos en distinta magnitud $\left({ }^{* *}\right)$. Finalmente, aunque existe una relación determinable entre los factores CC1 y DTO1, estas presentan estimadores no significativos. Por último en esta fase de modelamiento, la tabla 3 presenta los índices de bondad de ajuste del modelo propuesto que relaciona los factores de apoyo directivo, consecuencia de carrera y demandas de tiempo organización.

Se observa que los índices para el análisis de covarianza resultan confirmatorios. El índice CMIND/DF alcanza la magnitud de 2,587 inferior al valor máximo requerido $(\geq 3,0)$ al cual se agregan los indicadores de bondad de ajuste, IFI, TLI y CFI, que presentan valores por encima del requerido $(\geq 0,9)$. Complementariamente, el índice de error RMSA $(0,051)$ ratifica el modelo propuesto con un índice en torno al requerido $(\leq 0,05)$. Así mismo, respecto del análisis de varianza, los índices de bondad de ajuste resultan, en general, también confirmatorios. El índice CMIND/DF alcanzado es de 2,640 inferior al valor máximo esperado $(\geq 3,0)$ el cual se complementa con los índices de bondad de ajuste GFI, AGFI y CFI que presentan valores por encima y alrededor del requerido $(\geq 0,9)$. Por último el índice de error RMSA $(0,52)$ ratifica el modelo propuesto con un índice de bondad de ajuste en torno al requerido $(\leq 0,05)$. En consecuencia, apoyo directivo estructurado en dos factores (AP1; AP2), consecuencia de carrera en un solo factor (CC1) y demanda de tiempo organización (DTO1), resultan correctamente correlacionados estructurando un modelo de conciliación trabajo - familia.

Tabla 3: Índices de bondad de ajuste

\begin{tabular}{|c|c|c|c|c|c|c|c|c|c|}
\hline \multicolumn{10}{|c|}{ Análisis de covarianza } \\
\hline Modelo & CMIN & DF & $P$ & $\mathrm{CMIN} / \mathrm{I}$ & & GFI & AGFI & PGFI & \\
\hline Por defecto & 204,338 & 79 & 0,000 & 2,587 & & 0,959 & 0,938 & 0,631 & \\
\hline Saturado & 0,000 & 0 & & & & 1,000 & & & \\
\hline \multicolumn{6}{|c|}{ Comparaciones basales } & \multicolumn{4}{|c|}{ Medidas ajustadas de parsimonia } \\
\hline Modelo & NFI & RFI & $\mathrm{IFI}$ & TLI & CFI & PRATIO & PNFI & PCFI & \\
\hline Por defecto & 0,952 & 0,937 & 0,970 & 0,960 & 0,970 & 0,752 & 0,717 & 0,730 & \\
\hline Saturado & 1,000 & & 1,000 & & 1,000 & 0,000 & 0,000 & 0,000 & \\
\hline Modelo & RMSEA & LO 90 & $\mathrm{HI} 90$ & PCLOS: & & & & & \\
\hline \multicolumn{10}{|c|}{ Análisis de varianza } \\
\hline Modelo & CMIN & DF & $P$ & \multicolumn{2}{|c|}{ CMIN/DF } & GFI & AGFI & \multicolumn{2}{|c|}{ PGFI } \\
\hline Por defecto & 203,317 & 77 & 0,000 & 2,640 & & 0,959 & 0,937 & 0,616 & \\
\hline Saturado & 0,000 & 0 & & & & 1,000 & & & \\
\hline \multicolumn{6}{|c|}{ Comparaciones basales } & \multicolumn{4}{|c|}{ Medidas ajustadas de parsimonia } \\
\hline Modelo & NFI & RFI & IFI & TLI & CFI & PRATIO & PNFI & PCFI & \\
\hline Por defecto & 0,953 & 0,936 & 0,970 & 0,959 & 0,970 & 0,733 & 0,699 & 0,711 & \\
\hline Saturado & 1,000 & & 1,000 & & 1,000 & 0,000 & 0,000 & 0,000 & \\
\hline Modelo & RMSEA & LO 90 & $\mathrm{HI} 90$ & PCLOS & & & & & \\
\hline
\end{tabular}

\section{DISCUSIÓN}

El estudio de la relación trabajo - familia determinó los factores de apoyo directivo AP1 con seis reactivos y AP2 con tres ítems, explicando una varianza de $66,7 \%$ que evidencia el peso factorial de esta dimensión (Dawley et al., 2010) confirmando los factores determinados por McDonald et al., (2005). Por su parte consecuencia de carrera $\mathrm{CC} 1$ con sus tres componentes explica el $62,3 \%$ de la varianza y demanda de tiempo organización DTO1 con tres reactivos, explica el $69,8 \%$ de la varianza total, estructurando un constructo factorial coherente (Thompson et al., 1999; Pérez et al., 2017).

Así mismo, al relacionar los factores mediante covarianzas y varianzas se determinan índices significativos ratificando las vinculaciones determinadas por Warren y Johnson (1995), estableciendo incidencias mutuas (Allen, 2001; Amstad et al., 2011) dónde apoyo directivo funge como variable independiente y el factor de consecuencia de carrera se comporta como variable dependiente (Jansen et al., 2010), similar a la encontrada en los estudios de cultura familiar y bienestar (Lapierre y Allen, 2006; Allard et al., 2011; Martínez-Pampliega et al., 2019) que permiten a la organización convertirse en un lugar más agradable para trabajar, lo cual visibiliza la fuerza laboral y valida el rol de la dimensión consecuencia de carrera (Litchfield et al., 2004Mauno 
et al., 2005) propiciando la existencia de una política de vida laboral coherente y utilizable por parte de los personales (McDonald et al., 2005; Smith y Gardner, 2007, Jimenez-Figueroa y Bustamante, 2020).

A partir de los fundamentos de la conciliación e interfaz trabajo - familia (McMillan et al., 2011) que analiza los efectos de las conductas autónomas, de capacidad de decidir y de búsqueda del bienestar de los trabajadores (Litchfield et al., 2004; Thompson y Prottas, 2005), en el presente trabajo se observa que existen relaciones de covarianza y de varianzas inversas entre los factores de apoyo directivo AP1 $(-0,47$ y $-0,75)$ y AP2 $(-0,46$ y $-0,57)$ sobre consecuencias de carrera CC1, similar a los hallazgos presentados en los bloques jerárquicos determinados por Warren y Johnson (1995) que vinculó apoyo de los supervisores, tensión laboral y demandas de trabajo, limitando los efectos adversos y confirmando estudios equivalentes de conciliación trabajo-familia (Chinchilla et al., 2003; Jiménez et al., 2009; García, 2019) respecto de carreras profesionales tensas (Frye y Breaugh, 2004; Lapierre y Allen, 2006).

Como ha sido demostrado, el apoyo de los directivos estimula conductas de conciliación trabajo - familia en tanto propician la colaboración (Thompson et. al, 1999; Jimenez-Figueroa y Bustamante, 2020), en consecuencia, las relaciones así como las incidencias (covarianza y varianza) de apoyo directivo sobre consecuencia de carrera presentan estimadores equivalentes que ratifican los efectos (Dawley et al., 2010; Amstad et al., 2011), por una parte, porque pueden motivar a los personales y mejorar factores del clima organizacional (Allard et al., 2011; Bustamante y Álvarez, 2019) y por otra, porque gracias a la dirección facilitadora de jefaturas, la visibilización de la fuerza laboral disponible (Litchfield et al., 2004) y a la reducción de incertezas sobre consecuencias de carrera (McMillan et al., 2011; Sivatte y Gauadamillas, 2014) se logra una adecuada redistribución del tiempo de trabajo (Jansen et al., 2010) y con ello una mejor conciliación trabajo - familia (Thompson et. al, 1999; García, 2019).

Se determinó que el factor demanda de tiempo organización DTO1 impacta positivamente $(0,914$ y 1,099) potenciando los efectos y las percepciones positivas sobre las carreras de los personales (Sivatte y Gauadamillas, 2014), confirmando diversos hallazgos de la cultura trabajo-familia (Smith y Gardner, 2007; Pérez et al., 2017) y con ello, se logra validar que la conciliación de estos factores incrementa las percepciones de satisfacción laboral (Thompson et. al, 1999; Thompson y Prottas, 2005; Jiménez et al., 2009), en esta ocasión, de los personales del sector al por menor en Chile. En consecuencia, el factor demanda de tiempo organización DTO1, dado su origen en una política laboral determinada (McDonald et al., 2005) incide de manera fuerte sobre consecuencia de carrera, sin embargo, como ha sido estudiado, se requiere que los factores de apoyo directivo incidan sobre la estabilidad laboral, reduzcan la tensión interna y potencien los componentes de la organización y el desempeño como componentes del clima organizacional (Bustamante y Álvarez, 2019), en tanto fortalece las percepciones de seguridad de los empleados, incrementa el apego y el compromiso laborales (Casper y Harris, 2008) además de reducir el conflicto trabajo - familia y la cultura del trabajo y la familia (Mauno et al., 2005; Jimenez-Figueroa y Bustamante, 2020)

Verificando la hipótesis, se determina que los factores de apoyo directivo AP1 y AP2 se inciden mutuamente $(0,894$ y 0,914$)$ en términos de covarianzas y varianzas, de forma similar a los trabajos de Thompson et al., (1999) que ratificó dichos factores mediante sus alfas y varianzas explicadas. Además, se determinaron correlaciones inversas sobre consecuencia de carrera, por cuanto los personales de ambos géneros valoran una adecuada gestión de la interfaz trabajo - familia (McMillan et al., 2011), aunque presentan diferencias en los intereses y energías dedicadas al trabajo o a la familia a partir de los efectos moderadores de la demanda de tiempo del empleado en el trabajo y las demandas naturales de la paternidad, por una parte, porque prácticas directivas positivas permiten potenciar la vida familiar como lo señalan Jiménez et al., (2009) y como efecto cruzado, porque algunas prácticas de la familia inciden también en lo laboral (Chinchilla et al., 2003; Amstad et al., 2011; Martínez-Pampliega et al., 2019). Complementariamente el estrés y la falta de tiempo pueden llegar a generar efectos negativos sobre la conciliación trabajo - familia, especialmente relevante si se aprecian los cambios sociales y la composición demográfica de la fuerza laboral (Jimenez-Figueroa y Bustamante, 2020).

Sintetizando, una gestión eficaz de la conciliación trabajo - familia (Sivatte y Gauadamillas, 2014) mejora positivamente el bienestar de los empleados, fortalece la cultura trabajo- familia en la organización y estabiliza políticas empresariales de conciliación (McDonald et al., 2005). Se gestan entonces iniciativas de equilibrio produciendo un ciclo virtuoso digno de ser replicado (Smith y Gadner, 2007; Amstad et al., 2011; JimenezFigueroa y Bustamante, 2020). El factor independiente apoyo directivo resulta en consecuencia, ratificado en el modelamiento propuesto en este trabajo en la medida que incide de manera inversa sobre el bienestar organizacional reduciendo el impacto de consecuencia de carrera, lo cual resulta beneficioso si se realiza un adecuado afrontamiento centrado en el problema, como lo afirma Lapierre y Allen (2006). Finalmente, se ratifica que demanda de tiempo organización, incide en forma positiva y directa, con indicadores cercanos a la unidad, sobre consecuencia de carrera. 


\section{CONCLUSIONES}

Del análisis realizado, de la discusión de los resultados y de los hallazgos del presente trabajo se puede concluir que el modelamiento factorial permite ratificar los dos factores de apoyo directivo, el primero con seis y el segundo con tres reactivos, además de confirmar la estructura del factor de consecuencia de carrera que concentra tres ítems, seguido de la dimensión demanda de tiempo organización que incluye también tres reactivos, los cuales resultan confirmados mediante los índices de adecuación factorial, de bondad de ajuste y de confiabilidad respectivos. Se concluye que los fundamentos de conciliación trabajo - familia confirman $\mathrm{H} 1$ : de incidencia inversa de los factores de apoyo directivo sobre consecuencias de carrera y establece una relación alta y positiva de la demanda de tiempo organización sobre consecuencia de carrera lo que ubica el rol directivo en una posición de preeminencia, posicionando esta dimensión como un factor que influye sobre el bienestar organizacional.

Por último, considerando que el factor apoyo directivo es un factor relevante de la conciliación trabajo - familia y el factor demanda de tiempo organización actúa también sobre la dimensión consecuencia de carrera, la incidencia inversa de apoyo directivo y directa de demanda de tiempo organización sobre consecuencia de carrera, colabora fortaleciendo el compromiso organizacional así como potenciando las percepciones positivas de bienestar de los personales en sus respectivos espacios de trabajo.

\section{REFERENCIAS}

Allard, K., Hass, L., y Hwang C.P., Family-supportive organizational culture and fathers' experiences of work-family conflict in Sweden, https://doi.org/10.1111/j.1468-0432.2010.00540.x, Gender, Work \& Organization, 18(2), 141-157 (2011)

Allen, T., Family-supportive work environments: the role of organizational perceptions, https://doi.org/10.1006/jvbe.2000.1774, Journal of Vocational Behavior, 58(3), 414-435 (2001)

Amstad, F.T., Meier, L.L., y otros 3 autores, A meta-analysis of work-family conflict and various outcomes with a special emphasis on cross-domain versus matching-domain relations, https://doi.org/10.1037/a0022170, Journal of Occupational Health Psychology, 16(2), 151-169 (2011)

Bustamante, M.A., y Álvarez, A.J., Validación de un cuestionario de clima organizacional para organizaciones de salud, https://doi.org/10.11144/Javeriana.rgps18-36.vcco, Revista Gerencia y Políticas de Salud, 18(36) (2019)

Casper, W., Harris, C., Taylor-Bianco, A., y Holliday, J., Work-family conflict, perceived supervisor support and organizational commitment among Brazilian professionals, https://doi.org/10.1016/j.jvb.2011.04.011, Journal of Vocational Behavior, 79(3), 640-652 (2011)

Casper, W.J., y Harris, C.M., Work-life benefits and organizational attachment: self-interest utility and signaling theory models, https://doi.org/10.1016/j.jvb.2007.10.015, Journal of Vocational Behavior, 72(1), 95-109 (2008)

Chinchilla, M.N., Poelmans, S., y León, C., Políticas de conciliación trabajo-familia en 150 empresas españolas, Documento de investigación, Barcelona: IESE Business School, DI N498 Marzo (2003)

Clark, S., Work cultures and work/family balance, https://doi.org/10.1006/jvbe.2000.1759, Journal of Vocational Behavior, 58(3), 348-365 (2001)

Dawley, D., Hougton, J., y Bucklew, N., Perceived organizational support and turnover intention: the mediating effects of personal sacrifice and job fit, https://doi.org/10.1080/00224540903365463, Journal of Social Psychology, 150(3), 238$257(2010)$

Freiberg, H., Stover, J., y De la Iglesia, G., Fernández, M., Polychoric and tetrachoric correlations in exploratory and confirmatory factorial studies, Prensa Médica Latinoamericana Ciencias Psicológicas, 7(2), 151-164 (2013)

Frye, N., y Breaugh, J., Family-friendly policies, supervisor support, work-family conflict, family-work conflict, and satisfaction: a test of a conceptual model, https://doi.org/10.1007/s10869-004-0548-4, Journal of Business and Psychology, 19(2), 197-220 (2004)

García, B., El trabajo doméstico y de cuidado: su importancia y principales hallazgos en el caso mexicano, https://doi.org/10.24201/edu.v34i2.1811, Estudios Demográficos y Urbanos, 34(2), 237-267 (2019)

Hernández-Sampieri, R., y Mendoza, C., Metodología de la investigación, Las rutas cuantitativa, cualitativa y mixta, Editorial Mc Graw Hill Education, DF México, México (2018)

Jansen, N., Mohren, D., y otros tres autores, Changes in working time arrangements over time as a consequence of work-family conflict, https://doi.org/10.3109/07420528.2010.489874, Chronobiology International, 27(5), 1045-1061 (2010)

Jiménez, A., Acevedo, D., Salgado, A., y Moyano, E., Cultura trabajo- familia y compromiso organizacional en empresa de servicios, http://doi.org/10.1590/S1413-73722009000400013, Psicología em Estudo, 14(4), 729-738 (2009) 
Jimenez-Figueroa, A.E., y Bustamante, M.A., Directing support and career consequence in work-family conciliation, in the retail sector in Chile, https://dx.doi.org/10.4067/S0718-07642020000200063, Información Tecnológica, 31(2), 63-72 (2020)

Lapierre, L., y Allen, T., Work-supportive family, family.-supportive supervision, use of organizational benefits, and problem-focused coping: implications for work-family conflict and employee well-being, http://dx.doi.org/10.1037/10768998.11.2.169, Journal of Occupational Health Psychology, 11(2), 169-181 (2006)

Litchfield, L., Swanberg, J., y Sigworth, C., Increasing the visibility of the invisible workforce: model programs and policies for hourly and lower wage employees, Boston: Boston College Center for Work \& Family, Carroll School of Management April (2004)

Martínez-Pampliega, A., Ugarte, I., Merino, L., y Herrero, D., Conciliación familia-trabajo y sintomatología externalizante de los hijos e hijas: papel mediador del clima familiar, https://doi.org/10.23923/j.rips.2019.01.02, Revista Iberoamericana de Psicología y Salud, 10(1), 27-36 (2019)

Mauno, S., Kinnunen, U., y Pyykkö, M., Does work-family conflict mediate the relationship between work-family culture and self-reported distress? Evidence for five Finnish organizations, https://doi.org/10.1348/096317905X37082, Journal of Occupational and Organizational Psychology, 78(4), 509-530 (2005)

McDonald, P., Brown, K., y Bradley, L., Explanations for the provision-utilisation gap in work-life policy, https://doi.org/10.1108/09649420510579568, Women in Management Review, 20(1), 37-55 (2005)

McMillan, H., Morris, M., y Atchley, E., Constructs of the work/life interface: a synthesis of the literature and introduction of the concept of work/life harmony, https://doi.org/10.1177/1534484310384958, Human Resource Development Review, 10(1), 6-25 (2011)

Pérez, V., Palaci, F.J., y Topa, G., Cultura de conciliación y conflicto trabajo/familia en trabajadores con turnos laborales, https://doi.org/10.5944/ap.14.2.20024, Revista Acción Psicológica, Universidad Nacional de Educación a Distancia Madrid, España, 14(2), 193-120 (2017)

Sivatte, D., y Guadamillas, F., ¿La disponibilidad de medidas de conciliación y la cultura trabajo familia disminuyen el conflicto trabajo-familia de los empleados?, Universia Business Review, 4(44), 54-71 (2014)

Smith, J., y Gardner, D., Factors affecting employee use of work-life balance initiatives, https://doi.org/10.2307/30034926, New Zealand Journal of Psychology, 36(1), 3-12 (2007)

Thompson, C., Beauvais, L., y Lyness, K., When work-family benefits are not enough: the influence of work-family culture on benefit utilization, organizational attachment, and work-family conflict, https://doi.org/10.1006/jvbe.1998.1681, Journal of Vocational Behavior, 54(3), 392-415 (1999)

Thompson, C., y Prottas, D., Relationships among organizational family support, job autonomy, perceived control and employee well-being, https://doi.org/10.1037/1076-8998.10.4.100, Journal of Occupational Health Psychology, 10(4), 100-118 (2005)

Timmerman, M., y Lorenzo-Seva, U., Dimensionality assessment of ordered polytomous items with parallel analysis, https://doi.org/10.1037/a0023353, Psychological Methods, 16(2), 209-220 (2011)

Warren, J., y Johnson P., The impact of workplace support on work-family role strain, https://doi.org/10.2307/584803, Family Relations, 44(2), 163-169 (1995) 